\title{
Advances in glucose metabolism research in colorectal cancer (Review)
}

\author{
SITIAN FANG ${ }^{1,2}$ and XIAO FANG ${ }^{1,3}$ \\ ${ }^{1}$ Key Laboratory of Biotherapy of Zhejiang Province, Sir Run Run Shaw Hospital, Zhejiang University, \\ Hangzhou, Zhejiang 310016; ${ }^{2}$ Hangzhou No. 4 High School, Hangzhou, Zhejiang 310018; \\ ${ }^{3}$ Department of Anesthesiology, Sir Run Run Shaw Hospital, Zhejiang University, \\ Hangzhou, Zhejiang 310016, P.R. China
}

Received April 18, 2016; Accepted July 6, 2016

DOI: $10.3892 / b r .2016 .719$

\begin{abstract}
Cancer cells uptake glucose at a higher rate and produce lactic acid rather than metabolizing pyruvate through the tricarboxylic acid cycle. This adaptive metabolic shift is termed the Warburg effect. Recently progress had been made regarding the mechanistic understanding of glucose metabolism and associated diagnostic and therapeutic methods, which have been investigated in colorectal cancer. The majority of novel mechanisms involve important glucose metabolism associated genes and miRNA regulation. The present review discusses the contribution of these research results to facilitate with the development of novel diagnosis and anticancer treatment options.
\end{abstract}

Correspondence to: Dr Xiao Fang, Key Laboratory of Biotherapy of Zhejiang Province, Sir Run Run Shaw Hospital, Zhejiang University, 3 Qingchun East Road, Hangzhou, Zhejiang 310016, P.R. China

E-mail: fangx@srrsh.com

Abbreviations: TCA, tricarboxylic acid; FDG, fluorodeoxyglucose; $\mathrm{PET} / \mathrm{CT}$, positron emission tomography/computed tomography; HIF, hypoxia-inducible transcription factor; MDR, multidrug resistance; GRP78, glucose-regulated protein 78; ER, endoplasmic reticulum; Gluts, glucose transporters; DFS, disease-free survival; Glut3, glucose-transporter 3; AMPK, AMP-activated protein kinase; PrPc, prion protein; Glut1, glucose transporter 1; AREG, amphiregulin; drs-KO, drs-knockout; PDK1, pyruvate dehydrogenase kinase 1; PGI, phosphoglucose isomerase; TKTL1, transketolase-like 1; CAV1, caveolin 1; ChREBP, carbohydrate responsive element binding protein; mtDNA, mitochondrial DNA; SCO2, cytochrome $c$ oxidase 2; PKM, pyruvate kinase isozyme; LDHA, lactate dehydrogenase A; CRC, colorectal cancer; PPP, pentose phosphate pathway; TKT, transketolase; TKTL1, transketolase-like protein 1; FDG-PET, fluorodeoxyglucose positron emission tomography; CEA, carcinoembryonic antigen; MRI, magnetic resonance imaging; $\mathrm{QD}$, quantum dot; HF, halofuginone; ERMAs, energy-restriction mimetic agents; DCA, dichloroacetate; 2DG, 2-deoxy-D-glucose; ETCBs, electron transport chain blockers

Key words: glucose metabolism, colorectal cancer

\section{Contents}

1. Introduction

2. Novel mechanisms involving important glucose metabolism-associated genes

3. Numerous novel glucose metabolism mechanisms involve miRNA regulation

4. Advances in diagnostic and therapeutic methods associated with glucose metabolism

5. Conclusion

\section{Introduction}

Colorectal cancer (CRC) is the third leading cause of cancer-associated mortality in the world and the fourth most frequent type of cancer for men and women (1). Glucose consumption is required by living cells. Through glycolysis, glucose is mainly broken down into pyruvate, which enters into the tricarboxylic acid (TCA) cycle for maximum energy production. Cancer cells, however, uptake glucose at a higher rate and produce lactic acid, rather than metabolizing pyruvate through the TCA cycle. This adaptive metabolic shift is termed the Warburg effect, leading to anaerobic glycolysis, and is thought to provide an evolutionary advantage to cancer cells by providing increasing bioenergetics and biosynthesis (2). Overexpression of glucose transporters, such as glucose transporter (Glut) and a number of glycolytic enzymes, such as hexokinase (HK), lactate dehydrogenase (LDH) and pyruvate kinase (PK), contributes to the acquisition of this metabolic profile $(3,4)$.

Numerous proto-oncogenes (for example, Ras and c-Myc) and tumor suppressors (for example, p53) influence metabolism, and mutations in these genes upregulate glucose uptake in cancer cells and promote a metabolic phenotype, which supports tumor cell growth and proliferation. This is often mediated by oncogenic signaling pathways involving phosphoinositide 3-kinase (PI3K), hypoxia inducible factors (HIFs), p53 and v-myc avian myelocytomatosis viral oncogene homolog, which integrate nutrient and energy state signals to control the expression of genes important for concomitant cancer cell proliferation and energy metabolism (5).

Observation of elevated glucose uptake in cancer cells indicates the location of primary and metastatic tumor sites; 
for example, using F-18 fluorodeoxyglucose (FDG), a glucose analog, with a combination of positron emission tomography/computed tomography (PET/CT). Furthermore, there is a growing recognition that these metabolic changes may be exploited for targeted anticancer therapy. Identifying novel regulators of glucose metabolism in CRC would aid with the diagnosis, prognosis and future therapeutic interventions.

The current review presents recent advances in glucose metabolism research in CRC and discusses the contribution of these research results as a source of inspiration for developing novel diagnosis and anticancer treatment options.

\section{Novel mechanisms involving important glucose metabolism-associated genes}

The early expanding tumor usually outgrows the diffusion limits of its local blood supply, leading to hypoxia and stabilization of the hypoxia-inducible transcription factor, HIF. HIF initiates a transcriptional program that provides multiple solutions to hypoxic stress by regulating gene expression for tumor angiogenesis, glucose metabolism and resistance to oxidative stress. HIF-1 or HIF-2 are overexpressed and associated with patient prognosis in a variety of cancer types. However, previous data on HIF-1, HIF-2, and clinical outcome in CRC have been inconclusive, and all these studies were limited by small sample sizes $(n<136)(6-11)$. HIF-1A expression was demonstrated to be independently associated with a poor prognosis among 731 cases of CRC in two prospective cohort studies (12). Conversely, hypoxia-induced drug resistance is a major obstacle in the development of effective cancer therapeutic strategies. Wang et al (13) proposed that wogonin may be a promising candidate for the development of a novel multidrug resistance (MDR) reversal agent and that its reversal mechanism is probably due to the suppression of HIF-1A expression and glycolysis, via inhibiting the PI3K/Akt signaling pathway.

Heat shock protein glucose-regulated protein 78 (GRP78), an endoplasmic reticulum (ER) chaperone, is regulated by glucose (14). Proteomics combined with immunohistochemical analysis have demonstrated that the increased expression of GRP78 participates in the pathogenesis of colon cancer $(15,16)$. Therapy targeting for GRP78 may inhibit the formation of colon cancer tumors via the HIF-1A/vascular endothelial growth factor/VEGFR2 pathway.

Abnormally expressed Gluts are often co-localized with HIF-1A in peri-necrotic regions in human colorectal carcinoma. Huang et al (17) investigated signaling pathways of hypoxia-induced necroptosis and investigated the role of glucose pyruvate metabolite in mechanisms of death resistance. The authors found that the glycolytic metabolism confers resistance to necroptosis in hypoxic cancer cells partly through pyruvate scavenging of mitochondrial free radicals (17). Glut1 is the natural transporter of glucose and is required for the high glycolytic rate that is observed in colorectal tumors. Previous reports have noted that Glut1 overexpression in colorectal carcinomas is correlated with lymph node metastases and poor prognosis $(18,19)$. Furthermore, a tendency towards better disease-free survival (DFS), following long-course radiotherapy was noted if Glut1 staining intensity in the operative sample was negative or weak (20), indicating that Glut1 may serve as a hypoxic indicator for malignant potential in CRC patients.

Recently, an oncogenic function of yes-associated protein 1 (YAP; a downstream effector of the Hippo pathway) in promoting glycolysis and Glut3 was identified as a YAP-regulated gene involved in glucose metabolism. The Hippo pathway and AMP-activated protein kinase (AMPK) were activated during glucose starvation, resulting in phosphorylation of YAP and contributing to its inactivation, thereby providing a notable link between glucose metabolism and the Hippo pathway in tissue maintenance and cancer prevention (21).

Furthermore, cellular prion protein $(\mathrm{PrPc})$ is shown to be involved in regulating Glutl expression via the Fyn-HIF-2A pathway. PrPc is a glycosylphosphatidylinositol anchored membrane protein that has various physical functions, including protection against apoptotic and oxidative stress, cellular uptake of copper ions, transmembrane signaling, and adhesion to the extracellular matrix. Li et al (22) showed that PrPc is highly expressed in colorectal adenocarcinomas. Transcriptome profiling of PrPc-depleted DLD-1 cells revealed downregulation of Glut1 and silencing of PrPc inhibited tumorigenicity. This data characterized a novel molecular mechanism that links PrPc expression to the regulation of glycolysis (22).

In addition, Song et al (23) showed that astrocyte elevated gene-1 protein (AEG-1) may promote anaerobic glycolysis. A mechanistic insight into novel targets controlled by AEG-1 was presented and the authors indicated that components in the AEG-1/AMPK/phosphofructokinase 2 glycolysis process may be targeted for the clinical treatment of cancer (23).

It is well known that AMPK senses energetic changes and regulates glucose metabolism. Recently, Qiu et al (24) examined the mechanisms by which AMPK promotes metabolic adaptation in the tumor-bearing liver using a murine model of colon cancer liver metastasis. Knockout (KO) of AMPK $\alpha 2$ significantly enhanced tumor-induced glucose deprivation in the liver and increased the extent of liver injury and hepatocyte death. Mechanistically, AMPK $\alpha 2$ deficiency resulted in elevated reactive oxygen species, reduced mitophagy, and increased cell death in response to tumors or glucose deprivation in vitro. These results imply that AMPK $\alpha 2$ is essential for attenuation of liver injury during tumor metastasis via hepatic glucose deprivation and mitophagy-mediated inhibition of reactive oxygen species production. Therefore, AMPK $\alpha 2$ may represent an important therapeutic target for colon cancer metastasis-induced liver injury (24).

Amphiregulin (AREG), a member of the epidermal growth factor family and a rational target for CRC therapy, is essential for the three-dimensional structure of tumor formation. In CRC cells, glucose elevated the expression of Glut1 and AREG, as well as the activity of the HIF-1 luciferase reporter promoter. The suppression of AREG expression reduced the uptake of glucose and production of lactate. These data suggest that AREG is pivotal in the development of CRC via activation of the Warburg effect (25).

Previously, Tambe et al (26) demonstrated that the drs gene contributes to suppression of malignant tumor formation in drs-KO mice. The authors demonstrated that drs regulates glucose metabolism via lactate dehydrogenase-B (LDH-B). 
Downregulating drs may contribute to the Warburg effect, which is closely associated with malignant progression of cancer cells.

Bernatchez et al (27) reported that estrogen-related receptor $\alpha(E R R \alpha)$ depletion in colon cancer cells resulted in reduced glucose incorporation and glucose-mediated lipogenesis in these cells. ER R $\alpha$-depleted HCT116 cells displayed significant reduction in expression of a variety of key genes involved with glycolysis, the TCA cycle and lipid synthesis, such as hexokinase 1, glucose-6-phosphatase catalytic subunit, 6-phosphofructo-2-kinase/fructose-2,6-biphosphatase (PFKFB) 1, PFKFB2, aldolase C, fructose-bisphosphate, glutamic-pyruvic transaminase 2 and phosphoglucomutase 2 , likely reducing pyruvate production. These findings suggest that ERR $\alpha$ coordinates colon cancer cell proliferation and tumorigenic capacity via energy metabolism, and that ERR $\alpha$ may represent a promising therapeutic target in colon cancer.

Previously, the majority of the mechanism by which Wnt signaling drives proliferation during oncogenesis is attributed to its regulation of the cell cycle. Recently, Pate et al (28) identified an important mechanism by which Wnt signaling influenced Warburg metabolism. Pyruvate dehydrogenase kinase 1 (PDK1) was found to be an important direct target within a larger gene program for metabolism. PDK1 inhibits pyruvate flux to mitochondrial respiration and a rescue of its expression in Wnt-inhibited cancer cells rescues glycolysis, as well as vessel growth in the tumor microenvironment.

Transketolase-like 1 (TKTL1) induces glucose degradation via anaerobic pathways, even in presence of oxygen, favoring the malignant aerobic glycolytic phenotype characteristic of tumor cells. Tumor tissues from 63 patients diagnosed with $\mathrm{CRC}$ at different stages of progression were analyzed for TKTL1 by immunohistochemistry. Downregulation of this enzyme expression, and a correlation between enzyme expression and regional lymph-node involvement in colon cancer were detected when metastasis occurred. This finding may improve our understanding of metastasis and result in novel and more efficient therapeutic strategies against cancer (29).

Phosphoglucose isomerase (PGI) is a ubiquitous cytosolic enzyme that is key in glycolysis and gluconeogenesis pathways (30). Tsutsumi et al (31) determined that overexpression of PGI significantly contributes to the aggressive phenotype of human colon cancer and, thus, may provide a novel therapeutic target.

Another gene, caveolin 1 (CAV1) was also observed to enhance aerobic glycolysis in colon cancer cells via activation of solute carrier family 2 member 3/Glut 3 transcription. Depletion of elevated CAV1 levels reduces glucose uptake, intracellular ATP level and lactate accumulation, and triggers autophagy via activation of AMPK-TP53/p53 signaling (32). While Tong et al (33) demonstrated that the glucose responsive transcription factor carbohydrate responsive element binding protein is key in redirecting glucose metabolism to anabolic pathways and suppressing p53 activity.

\section{Numerous novel glucose metabolism mechanisms involve miRNA regulation}

Genome instability is regarded as a hallmark of cancer. Human tumors frequently carry clonally expanded mutations in their mitochondrial DNA (mtDNA), some of which may drive cancer progression and metastasis. Ericson et al (34) directly measured the frequency of nonclonal (random) de novo single base substitutions in the mtDNA of human CRCs. Notably, tumor tissue exhibited a decreased prevalence of these mutations relative to the adjacent non-tumor tissue. The difference in mutation burden was attributable to a reduction in $\mathrm{C}: \mathrm{G}$ to $\mathrm{T}$ :A transitions, which are associated with oxidative damage. The study demonstrated that the lower random mutation frequency in tumor tissue was also coupled with a shift in glucose metabolism from oxidative phosphorylation to anaerobic glycolysis, as compared to non-neoplastic colon tissue. Together these findings indicate that the fidelity of the mitochondrial genome is increased in cancer as a result of a decrease in reactive oxygen species-mediated mtDNA damage.

Tumor progression is driven by genetic mutations; however, little is known about the environmental conditions that select for these mutations. Investigating the transcriptomes of paired CRC cell lines that differed only in the mutational status of their KRAS proto-oncogene, GTPase (KRAS) or B-Raf proto-oncogene, serine/threonine kinase (BRAF) genes, Yun et al (35) found that GLUT1 was one of three genes consistently upregulated in cells with KRAS or BRAF mutations. The mutant cells exhibited enhanced glucose uptake and glycolysis, and survived in low glucose conditions. All the phenotypes that require GLUT1 expression suggest that glucose deprivation drives the acquisition of KRAS pathway mutations in human tumors.

Conversely, the pro-inflammatory cytokines, tumor necrosis factor- $\alpha(\mathrm{TNF} \alpha)$ and interleukin (IL)-17 may stimulate glycolysis and growth factor production in CRC cells (36). Necrosis factor $-\kappa \mathrm{B}(\mathrm{NF}-\kappa \mathrm{B})$ organizes energy metabolism networks by controlling the balance between the utilization of glycolysis and mitochondrial respiration. NF- $\mathrm{kB}$ inhibition causes cellular reprogramming to aerobic glycolysis under basal conditions and induces necrosis on glucose starvation. This NF-кB-dependent metabolic pathway involves stimulation of oxidative phosphorylation through upregulation of mitochondrial synthesis of cytochrome $c$ oxidase 2 (37).

However, the majority of recent studies have shown that in cancer cells the alterations of glucose metabolism are associated with miRNA dysregulation $(38,39)$. miRNAs participate in cell metabolism by regulating the expression of genes whose protein products either directly regulate metabolic machinery or indirectly modulate the expression of metabolic enzymes, serving as master regulators (40). For example, miR-26a regulates glucose metabolism of CRC cells by directly targeting the pyruvate dehydrogenase complex component X (PDHX), which inhibits the conversion of pyruvate to acetyl coenzyme A in the citric acid cycle (41).

The overexpression of hexokinase 2 (HK2) in cancer is thought to provide cancer cells with a growth advantage due to increased glycolytic flux, by promoting the first step of glycolysis and thus promoting/inducing the shift towards aerobic glycolysis. Gregersen et al (42) identified that HK2 affects glucose metabolism in colon cancer cells and validated HK2 as an miR-143 target. Loss of miR-143-mediated repression of HK2 promotes glucose metabolism in cancer cells, contributing to the shift towards aerobic glycolysis that is observed in many types of tumor. 
Sun et al (43) reported that miR-124 alters the expression of genes involved in glucose metabolism and stimulates cancer cell apoptosis. In addition, the positive feedback loop between miR-124 and pyruvate kinase isozymes M1 (PKM1)/hepatocyte nuclear factor $4 \alpha(\mathrm{HNF} 4 \alpha)$ is important in CRC cell apoptosis; indicating that disrupting this regulatory circuit might be a potential therapeutic tool for CRC treatment. In addition, Sun et al (44) showed that miR-124, miR-137 and miR-340 are associated with poor prognosis of CRC. Expression of these miRNAs inhibits the growth of colorectal cancer cells. miR-124, miR-137 and miR-340 target PKM alternative splicing proteins [polypyrimidine tract-binding protein 1 (PTBP1)/heterogeneous nuclear ribonucleoprotein (hnRNAP) A1/hnRNAPA2], which control the inclusion of exon 9 (PKM1) or exon 10 (PKM2). Consequently, miR-124, miR-137 and miR-340 switch the PKM gene expression from PKM2 to PKM1. High ratios of PKM1/PKM2 inhibit the glycolysis rate, but elevate the glucose flux into oxidative phosphorylation. These results demonstrate that miRNAs (miR-124, miR-137 and miR-340) impair CRC growth by counteracting the Warburg effect due to regulating alternative splicing of the PKM gene.

A previous study has shown that higher expression levels of LDHA are associated with CRC. Recently, Wang et al (45) investigated miRNA-induced changes in LDHA expression. Knockdown of LDHA resulted in decreased lactate and ATP production, and glucose uptake. CRC cells with knockdown of LDHA exhibited markedly slower growth rates than control cells. Furthermore, it was found that miR-34a, miR-34c, miR-369-3p, miR-374a, and miR-4524a/b target LDHA and regulate glycolysis in cancer cells (45). In addition, the authors also identified a genetic loci newly associated with increased CRC progression, rs 18407893 at 11p15.4 (in the 3'-untranslated region of LDHA), which maps to the seed sequence recognized by miR-374a. Cancer cells overexpressing miR-374a exhibit decreased levels of LDHA when compared with miR-374a-mutation (rs18407893 at 11p15.4).

miRNAs have been proposed to be important in tumorigenesis and drug resistance in colon cancer. miR-26a regulates glucose metabolism of CRC cells by directly targeting PDHX, which inhibits the conversion of pyruvate to acetyl coenzyme A in the citric acid cycle (41). He et al (46) reported that overexpression of miR-122 in fluorouracil (5-FU)-resistant cells resensitizes 5-FU resistance through the inhibition of PKM2 in vitro and in vivo. Furthermore, glucose metabolism is significantly upregulated in 5-FU-resistant cells.

Additionally, Li et al (47) found that miRNAs are significant in 5-FU-resistant colon cancer cells. The cells exhibited upregulation of LDHA expression and activity compared with parental cells. LDHA was also shown to be a direct target of miR-34a. This indicates that miR-34a-mediated inhibition of glucose metabolism may present as a therapeutic target in patients with chemoresistant colon cancer.

Ellis et al (48) reported that colorectal neoplasia differentially expressed (CRNDE), an lncRNA, was regulated by insulin/insulin-like growth factors, and their findings indicate a role for CRNDE nuclear transcripts in regulating cellular metabolism, including glucose and lipid metabolism, which may correlate with their upregulation in CRC.
Various studies strongly suggested that miR-124 and polypyrimidine tract binding protein 1 (PTBP1) have the potential to be target molecules for the development of anticancer medication, as they are able to negatively affect the entire energy metabolism process that specifically works in cancer cells $(44,49,50)$.

Alteration of glucose metabolism in cancer influences various important metabolic pathways including the pentose phosphate pathway (PPP). PPP allows glucose conversion to ribose for nucleic acid synthesis, glucose degradation to lactate and regeneration of redox equivalents. The non-oxidative aspect of the PPP is controlled by transketolase (TKT) enzymes. Xu et al (51) provided intimated the importance of the TKT-like 1 (TKTL1; one TKT isoform) dysregulation in human HCT116 colon carcinoma cells and indicated that TKTL1 overexpression may be considered as a novel tumor marker, as well as a promising target for anticancer therapy. Furthermore, Shibuya et al (52) reported that the synthesis of glycolytic and PPP enzymes is almost ubiquitously augmented in colorectal carcinoma specimens and that pharmacological inhibition of the mechanistic target of rapamycin (mTOR)-PPP axis presents a promising therapeutic strategy against CRCs.

In addition, Ma et al (53) showed that protein kinase $\mathrm{C} \zeta(\mathrm{PKC} \zeta)$ deficiency promotes the plasticity that is necessary for cancer cells to reprogram their metabolism to utilize glutamine via the serine biosynthetic pathway in the absence of glucose. PKC $\zeta$ represses the expression of two key enzymes of the pathway, D-3-phosphoglycerate dehydrogenase (PHGDH) and phosphoserine aminotransferase 1 , and phosphorylates PHGDH at key residues to inhibit its enzymatic activity. The loss of PKC $\zeta$ in mice results in enhanced intestinal tumorigenesis and increased levels of these two metabolic enzymes, whereas patients with low levels of $\mathrm{PKC} \zeta$ have a poor prognosis, suggesting that $\mathrm{PKC} \zeta$ is a critical metabolic tumor suppressor in mouse and human cancer.

\section{Advances in diagnostic and therapeutic methods associated with glucose metabolism}

It is well known that carcinoembryonic antigen (CEA) detection in the serum and tumor tissue of CRC patients is the most commonly used marker for the diagnosis and evaluation of prognosis or recurrence following treatment; however, its role remains controversial. Previous studies have demonstrated that the sensitivity of CEA is only $30 \%$ (54). The Warburg effect has since been demonstrated in different types of tumors and the concomitant increase in glucose uptake has been exploited clinically for the detection of tumors by fluorodeoxyglucose (FDG) positron emission tomography (PET). It should be emphasized that there are potential limitations when imaging CRC patients by FDG-PET. False-positive FDG uptake is well recognized and may be problematic, with various studies reporting that between 5 and $8 \%$ of CRC patients may be falsely upstaged by PET-computed tomography (CT) when detecting disease recurrence. Future potential applications include the use of 'one-stop shop' imaging with contrastenhanced PET-CT, integration of novel PET tracers, and the advent of PET-magnetic resonance imaging (MRI); all of which may find increasing clinical application in the assessment and management of CRC patients (55). 
Xing et al (56) developed a unique cell-targeted, paramagnetic-fluorescent double-signal molecular nanoprobe for CRC in vivo MRI diagnosis and subsequent biopsy. The unique molecular nanoprobe is composed of a fluorescent quantum dot (QD) core; a coating layer of paramagnetic diethylenetriaminepentaacetic acid (DTPA)-gadolinium (Gd) coupled bovine serum albumin (BSA; GdDTPA-BSA) and a surface targeting moiety of anti-Glut1 polyclonal antibody (PcAb). The engineered GdDTPA·BSA@QDs-PcAb is $35 \mathrm{~nm}$ in diameter and colloidally stable under basic and acidic conditions.

Metabolic reprogramming resulting in enhanced glycolysis is a phenotypic trait of cancer cells, which is imposed by the tumor microenvironment and is linked to the downregulation of the catalytic subunit of the mitochondrial $\mathrm{H}^{+}$-ATPase (b-F1-ATPase). The bioenergetic signature is a protein ratio (b-F1-ATPase/GAPDH), which provides an estimate of glucose metabolism in tumors and serves as a prognostic indicator for cancer patients. Sánchez-Aragó and Cuezva (57) suggested that the determination of this bioenergetic signature of colon carcinomas could provide a tool for predicting the therapeutic response to various chemotherapeutic strategies aimed at combating tumor progression.

The growing interest in utilizing energy restriction without malnutrition as an approach for cancer therapy and prevention is based on the promising results from animal and human trials $(58,59)$. The principle of targeting energy metabolism as an antitumor strategy relies on the differences in the mechanisms by which normal and transformed cells generate energy (5). Therefore, cancer cells are particularly vulnerable to energy restriction. Halofuginone treatment inhibits CRC growth in vitro and in vivo by regulating the Akt/mTORC1 signaling pathway to inhibit glucose uptake and glycolysis in CRC cells (60).

Energy-restriction mimetic agents (ERMAs) are small-molecule agents that target various aspects of energy metabolism.ERMAs have emerged as a promising approach for cancer therapy. OSU-CG5, a novel ERMA, exhibits promising anticancer activity against human CRC cells in vitro, which was, at least in part, due to energy restriction and the consequent induction of ER stress and apoptosis (61). Inhibition of PDK by dichloroacetate (DCA) shifts tumor cell metabolism from anaerobic glycolysis to glucose oxidation, with activation of mitochondrial activity and chemotherapy-dependent apoptosis (62). The ability of an inhibitor of glucose metabolism, 2-deoxy-D-glucose (2DG), combined with mitochondrial electron transport chain blockers (ETCBs) to enhance oxidative stress and cytotoxicity was also determined in human colon cancer cells (63). Furthermore, a novel mechanism involves selenium-binding protein 1 mediating cancer inhibition via altering lipid/glucose metabolic signaling pathways (64). In addition, selective inhibition of glucose-6-phosphate dehydrogenase may represent an important mechanism by which aspirin may exert its anticancer effects through inhibition of ribonucleotide synthesis (65).

\section{Conclusion}

Recently, progress had been made regarding the mechanistic understanding of glucose metabolism and associated diagnostic and therapeutic methods in CRC. The majority of novel mechanisms involved important glucose metabolismassociated genes and miRNA regulation. Therefore, from a therapeutic perspective, the mechanistic understanding of glucose metabolism in cellular regulation will enable the identification of novel therapeutic targets and will facilitate the design of metabolite mimetics, which are uniquely taken up by cancer cells or converted into the active form by enzymes that are upregulated in tumors. Profiling of signaling pathways that are affected in glucose metabolism may also allow the development of diagnostic tests of cancer. In addition, metabolite derivatives may be used for the molecular imaging and classification of cancer. However, the present review emphasizes the requirement to investigate beyond glucose metabolism to a broader study, which encompasses all of the metabolic requirements of a cancer cell.

\section{Acknowledgements}

The present study was supported by grants from the National Natural Science Foundation of China (grant nos. 81372622 and 81472211) and Major Projects in Zhejiang Province (grant no. 2012C13014-1).

\section{References}

1. Arnold M, Sierra MS, Laversanne M, Soerjomataram I, Jemal A and Bray F: Global patterns and trends in colorectal cancer incidence and mortality. Gut: Jan 27, 2016 (Epub ahead of print). doi: 10.1136/gutjnl-2015-310912.

2. Cerella C, Gaigneaux A,Dicato M and Diederich M: Antagonistic role of natural compounds in mTOR-mediated metabolic reprogramming. Cancer Lett 356: 251-262, 2015.

3. Cerella C, Radogna F, Dicato M and Diederich M: Natural compounds as regulators of the cancer cell metabolism. Int J Cell Biol 2013: 639401, 2013.

4. Cerella C, Michiels C, Dashwood RH, Surh YJ and Diederich M: Metabolism and cancer: old and new players. Int J Cell Biol 2013: 293201, 2013

5. Cairns RA, Harris IS and Mak TW: Regulation of cancer cell metabolism. Nat Rev Cancer 11: 85-95, 2011.

6. Rasheed S, Harris AL, Tekkis PP, Turley H, Silver A, McDonald PJ, Talbot IC, Glynne-Jones R, Northover JM and Guenther T: Hypoxia-inducible factor-1alpha and -2alpha are expressed in most rectal cancers but only hypoxia-inducible factor-1alpha is associated with prognosis. Br J Cancer 100: 1666-1673, 2009.

7. Yoshimura H, Dhar DK, Kohno H, Kubota H, Fujii T, Ueda S, Kinugasa S, Tachibana M and Nagasue N: Prognostic impact of hypoxia-inducible factors 1alpha and 2alpha in colorectal cancer patients: correlation with tumor angiogenesis and cyclooxygenase-2 expression. Clin Cancer Res 10: 8554-8560, 2004.

8. Schmitz KJ, Müller CI, Reis H, Alakus H, Winde G, Baba HA Wohlschlaeger J, Jasani B, Fandrey J and Schmid KW: Combined analysis of hypoxia-inducible factor 1 alpha and metallothionein indicates an aggressive subtype of colorectal carcinoma. Int J Colorectal Dis 24: 1287-1296, 2009.

9. Rajaganeshan R, Prasad R, Guillou PJ, Scott N, Poston G and Jayne DG: Expression patterns of hypoxic markers at the invasive margin of colorectal cancers and liver metastases. Eur J Surg Oncol 35: 1286-1294,2009.

10. Kuwai T, Kitadai Y, Tanaka S, Onogawa S, Matsutani N, Kaio E, Ito $M$ and Chayama $\mathrm{K}$ : Expression of hypoxia-inducible factor-1alpha is associated with tumor vascularization in human colorectal carcinoma. Int J Cancer 105: 176-181, 2003.

11. Furlan D, Sahnane N, Carnevali I, Cerutti R, Bertoni F, Kwee I, Uccella S, Bertolini V, Chiaravalli AM and Capella C: Up-regulation of the hypoxia-inducible factor-1 transcriptional pathway in colorectal carcinomas. Hum Pathol 39: 1483-1494, 2008

12. Baba Y, Nosho K, Shima K, Irahara N, Chan AT, Meyerhardt JA, Chung DC, Giovannucci EL, Fuchs CS and Ogino S: HIF1A overexpression is associated with poor prognosis in a cohort of 731 colorectal cancers. Am J Pathol 176: 2292-2301, 2010. 
13. Wang H, Zhao L, Zhu LT, Wang Y, Pan D, Yao J, You QD and Guo QL: Wogonin reverses hypoxia resistance of human colon cancer HCT116 cells via downregulation of HIF-1 $\alpha$ and glycolysis, by inhibiting PI3K/Akt signaling pathway. Mol Carcinog 53 (Suppl 1): E107-E118, 2014.

14. Kozutsumi Y, Segal M, Normington K, Gething MJ and Sambrook J: The presence of malfolded proteins in the endoplasmic reticulum signals the induction of glucose-regulated proteins. Nature 332: 462-464, 1988.

15. Xing X, Lai M, Wang Y, Xu E and Huang Q: Overexpression of glucose-regulated protein 78 in colon cancer. Clin Chim Acta 364: 308-315, 2006.

16. Takahashi H, Wang JP, Zheng HC, Masuda S and Takano Y: Overexpression of GRP78 and GRP94 is involved in colorectal carcinogenesis. Histol Histopathol 26: 663-671, 2011.

17. Huang CY, Kuo WT, Huang YC, Lee TC and Yu LC: Resistance to hypoxia-induced necroptosis is conferred by glycolytic pyruvate scavenging of mitochondrial superoxide in colorectal cancer cells. Cell Death Dis 4: e622, 2013.

18. Younes M, Lechago LV and Lechago J: Overexpression of the human erythrocyte glucose transporter occurs as a late event in human colorectal carcinogenesis and is associated with an increased incidence of lymph node metastases. Clin Cancer Res 2: 1151-1154, 1996.

19. Haber RS, Rathan A, Weiser KR, Pritsker A, Itzkowitz SH, Bodian C, Slater G, Weiss A and Burstein DE: GLUT1 glucose transporter expression in colorectal carcinoma: A marker for poor prognosis. Cancer 83: 34-40, 1998.

20. Korkeila E, Jaakkola PM, Syrjänen K, Pyrhönen S and Sundström J: Pronounced tumour regression after radiotherapy is associated with negative/weak glucose transporter-1 expression in rectal cancer. Anticancer Res 31: 311-315, 2011

21. Wang W, Xiao ZD, Li X, Aziz KE, Gan B, Johnson RL and Chen J: AMPK modulates Hippo pathway activity to regulate energy homeostasis. Nat Cell Biol 17: 490-499, 2015.

22. Li QQ, Sun YP, Ruan CP, Xu XY, Ge JH, He J, Xu ZD, Wang Q and Gao WC: Cellular prion protein promotes glucose uptake through the Fyn-HIF-2 $\alpha$-Glut1 pathway to support colorectal cancer cell survival. Cancer Sci 102: 400-406, 2011

23. Song HT, Qin Y, Yao GD, Tian ZN, Fu SB and Geng JS: Astrocyte elevated gene-1 mediates glycolysis and tumorigenesis in colorectal carcinoma cells via AMPK signaling. Mediators Inflamm 2014: 287381, 2014

24. Qiu SL, Xiao ZC, Piao CM, Xian YL, Jia LX, Qi YF, Han JH, Zhang YY and Du J: AMP-activated protein kinase $\alpha 2$ protects against liver injury from metastasized tumors via reduced glucose deprivation-induced oxidative stress. J Biol Chem 289 9449-9459, 2014.

25. Nam SO, Yotsumoto F, Miyata K, Fukagawa S, Yamada H, Kuroki M and Miyamoto S: Warburg effect regulated by amphiregulin in the development of colorectal cancer. Cancer Med 4 $575-587,2015$

26. Tambe Y, Hasebe M, Kim CJ, Yamamoto A and Inoue H: The drs tumor suppressor regulates glucose metabolism via lactate dehydrogenase-B. Mol Carcinog 55: 52-63, 2016.

27. Bernatchez G, Giroux V, Lassalle T, Carpentier AC, Rivard N and Carrier JC: ERR $\alpha$ metabolic nuclear receptor controls growth of colon cancer cells. Carcinogenesis 34: 2253-2261, 2013.

28. Pate KT, Stringari C, Sprowl-Tanio S, Wang K, TeSlaa T, Hoverter NP, McQuade MM, Garner C, Digman MA, Teitell MA, et al: Wnt signaling directs a metabolic program of glycolysis and angiogenesis in colon cancer. EMBO J 33 1454-1473, 2014

29. Diaz-Moralli S, Tarrado-Castellarnau M, Alenda C, Castells A and Cascante M: Transketolase-like 1 expression is modulated during colorectal cancer progression and metastasis formation. PLoS One 6: e25323, 2011

30. Harrison RA: The detection of hexokinase, glucosephosphate isomerase and phosphoglucomutase activities in polyacrylamide gels after electrophoresis: a novel method using immobilized glucose 6-phosphate dehydrogenase. Anal Biochem 61: 500-507, 1974

31. Tsutsumi S, Fukasawa T, Yamauchi H, Kato T, Kigure W, Morita H, Asao T and Kuwano H: Phosphoglucose isomerase enhances colorectal cancer metastasis. Int J Oncol 35: $1117-1121,2009$

32. Ha TK and Chi SG: CAV1/caveolin 1 enhances aerobic glycolysis in colon cancer cells via activation of SLC2A3/GLUT3 transcription. Autophagy 8: 1684-1685, 2012
33. Tong X, Zhao F, Mancuso A, Gruber JJ and Thompson CB: The glucose-responsive transcription factor ChREBP contributes to glucose-dependent anabolic synthesis and cell proliferation. Proc Natl Acad Sci USA 106: 21660-21665, 2009.

34. Ericson NG, Kulawiec M, Vermulst M, Sheahan K, O'Sullivan J, Salk JJ and Bielas JH: Decreased mitochondrial DNA mutagenesis in human colorectal cancer. PLoS Genet 8: e1002689, 2012.

35. Yun J, Rago C, Cheong I, Pagliarini R, Angenendt P, Rajagopalan H, Schmidt K, Willson JK, Markowitz S, Zhou S, et al: Glucose deprivation contributes to the development of KRAS pathway mutations in tumor cells. Science 325: $1555-1559,2009$

36. Straus DS: TNF $\alpha$ and IL-17 cooperatively stimulate glucose metabolism and growth factor production in human colorectal cancer cells. Mol Cancer 12: 78, 2013.

37. Mauro C, Leow SC, Anso E, Rocha S, Thotakura AK, Tornatore L, Moretti M, De Smaele E, Beg AA, Tergaonkar V, et al: NF- $\kappa B$ controls energy homeostasis and metabolic adaptation by upregulating mitochondrial respiration. Nat Cell Biol 13. 1272-1279, 2011.

38. Pucci S and Mazzarelli P: MicroRNA dysregulation in colon cancer microenvironment interactions: the importance of small things in metastases. Cancer Microenviron 4: 155-162, 2011.

39. Fang R, Xiao T, Fang Z, Sun Y, Li F, Gao Y, Feng Y, Li L, Wang Y, Liu X, et al: MicroRNA-143 (miR-143) regulates cancer glycolysis via targeting hexokinase 2 gene. J Biol Chem 287: 23227-23235, 2012.

40. Singh PK, Brand RE and Mehla K: MicroRNAs in pancreatic cancer metabolism. Nat Rev Gastroenterol Hepatol 9: 334-344, 2012

41. Chen B, Liu Y, Jin X, Lu W, Liu J, Xia Z, Yuan Q, Zhao X, Xu N and Liang S: MicroRNA-26a regulates glucose metabolism by direct targeting PDHX in colorectal cancer cells. BMC Cancer 14: 443, 2014.

42. Gregersen LH, Jacobsen A, Frankel LB, Wen J, Krogh A and Lund AH: MicroRNA-143 down-regulates Hexokinase 2 in colon cancer cells. BMC Cancer 12: 232, 2012.

43. Sun Y, Zhao X, Luo M, Zhou Y, Ren W, Wu K, Li X, Shen J and Hu Y: The pro-apoptotic role of the regulatory feedback loop between miR-124 and PKM1/HNF4 $\alpha$ in colorectal cancer cells. Int J Mol Sci 15: 4318-4332, 2014

44. Sun Y, Zhao X, Zhou Y and Hu Y: miR-124, miR-137 and miR-340 regulate colorectal cancer growth via inhibition of the Warburg effect. Oncol Rep 28: 1346-1352, 2012.

45. Wang J, Wang H, Liu A, Fang C, Hao J and Wang Z: Lactate dehydrogenase A negatively regulated by miRNAs promotes aerobic glycolysis and is increased in colorectal cancer. Oncotarget 6 : 19456-19468, 2015.

46. He J, Xie G, Tong J, Peng Y, Huang H, Li J, Wang N and Liang H: Overexpression of microRNA-122 re-sensitizes 5-FU-resistant colon cancer cells to 5-FU through the inhibition of PKM2 in vitro and in vivo. Cell Biochem Biophys 70: 1343-1350, 2014.

47. Li X, Zhao H, Zhou X and Song L: Inhibition of lactate dehydrogenase A by microRNA-34a resensitizes colon cancer cells to 5-fluorouracil. Mol Med Rep 11: 577-582, 2015.

48. Ellis BC, Graham LD and Molloy PL: CRNDE, a long non-coding RNA responsive to insulin/IGF signaling, regulates genes involved in central metabolism. Biochim Biophys Acta 1843: 372-386, 2014.

49. Taniguchi K, Sugito N, Kumazaki M, Shinohara H, Yamada N, Nakagawa Y, Ito Y, Otsuki Y, Uno B, Uchiyama K, et al: MicroRNA-124 inhibits cancer cell growth through PTB1/PKM1/PKM2 feedback cascade in colorectal cancer. Cancer Lett 363: 17-27, 2015.

50. Taniguchi K, Sugito N, Kumazaki M, Shinohara H, Yamada N, Matsuhashi N, Futamura M, Ito Y, Otsuki Y, Yoshida K, et al: Positive feedback of DDX6/c-Myc/PTB1 regulated by miR-124 contributes to maintenance of the Warburg effect in colon cancer cells. Biochim Biophys Acta 1852: 1971-1980, 2015.

51. Xu X, Zur Hausen A, Coy JF and Löchelt M: Transketolase-like protein 1 (TKTL1) is required for rapid cell growth and full viability of human tumor cells. Int J Cancer 124: 1330-1337, 2009.

52. Shibuya N, Inoue K, Tanaka G, Akimoto K and Kubota K: Augmented pentose phosphate pathway plays critical roles in colorectal carcinomas. Oncology 88: 309-319, 2015.

53. Ma L, Tao Y, Duran A, Llado V, Galvez A, Barger JF, Castilla EA, Chen J, Yajima T, Porollo A, et al: Control of nutrient stress-induced metabolic reprogramming by PKC $\zeta$ in tumorigenesis. Cell 152: 599-611, 2013.

54. Duffy MJ: Carcinoembryonic antigen as a marker for colorectal cancer: Is it clinically useful? Clin Chem 47: 624-630, 2001. 
55. Culverwell AD, Chowdhury FU and Scarsbrook AF: Optimizing the role of FDG PET-CT for potentially operable metastatic colorectal cancer. Abdom Imaging 37: 1021-1031, 2012.

56. Xing X, Zhang B, Wang X, Liu F, Shi D and Cheng Y: An 'imaging-biopsy' strategy for colorectal tumor reconfirmation by multipurpose paramagnetic quantum dots. Biomaterials 48: $16-25,2015$.

57. Sánchez-Aragó M and Cuezva JM: The bioenergetic signature of isogenic colon cancer cells predicts the cell death response to treatment with 3-bromopyruvate, iodoacetate or 5-fluorouracil. J Transl Med 9: 19, 2011.

58. Omar HA, Berman-Booty L and Weng JR: Energy restriction: stepping stones towards cancer therapy. Future Oncol 8 : 1503-1506, 2012.

59. Hursting SD, Dunlap SM, Ford NA, Hursting MJ and Lashinger LM: Calorie restriction and cancer prevention: A mechanistic perspective. Cancer Metab 1: 10, 2013.

60. Chen GQ, Tang CF, Shi XK, Lin CY, Fatima S, Pan XH, Yang DJ, Zhang G, Lu AP, Lin SH, et al: Halofuginone inhibits colorectal cancer growth through suppression of Akt/mTORC1 signaling and glucose metabolism. Oncotarget 6: 24148-24162, 2015.
61. Arafa SA, Abdelazeem AH, Arab HH and Omar HA: OSU-CG5, a novel energy restriction mimetic agent, targets human colorectal cancer cells in vitro. Acta Pharmacol Sin 35: 394-400, 2014.

62. Zwicker F, Kirsner A, Peschke P, Roeder F, Debus J, Huber PE and Weber KJ: Dichloroacetate induces tumor-specific radiosensitivity in vitro but attenuates radiation-induced tumor growth delay in vivo. Strahlenther Onkol 189: 684-692, 2013.

63. Fath MA, Diers AR, Aykin-Burns N, Simons AL, Hua L and Spitz DR: Mitochondrial electron transport chain blockers enhance 2-deoxy-D-glucose induced oxidative stress and cell killing in human colon carcinoma cells. Cancer Biol Ther 8: 1228-1236, 2009.

64. Ying Q, Ansong E, Diamond AM, Lu Z, Yang W and Bie X: Quantitative proteomic analysis reveals that anti-cancer effects of selenium-binding protein 1 in vivo are associated with metabolic pathways. PLoS One 10: e0126285, 2015.

65. Marimuthu S, Chivukula RS, Alfonso LF, Moridani M, Hagen FK and Bhat GJ: Aspirin acetylates multiple cellular proteins in HCT-116 colon cancer cells: identification of novel targets. Int J Oncol 39: 1273-1283, 2011. 